\title{
Evaluation of inflammation-based markers for predicting the prognosis of unresectable pancreatic ductal adenocarcinoma treated with chemotherapy
}

\author{
HIROYUKI ASAMA $^{1}$, REI SUZUKI ${ }^{1}$, TADAYUKI TAKAGI ${ }^{1}$, MITSURU SUGIMOTO ${ }^{1}$, \\ NAOKI KONNO $^{1}$, KO WATANABE ${ }^{1,2}$, JUN NAKAMURA ${ }^{1,2}$, HITOMI KIKUCHI $^{1,2}$, MIKA TAKASUMI ${ }^{1}$, \\ YUKI SATO $^{1}$, HIROKI IRIE ${ }^{1}$, TAKUTO HIKICHI ${ }^{2}$ and HIROMASA OHIRA ${ }^{1}$ \\ Departments of ${ }^{1}$ Gastroenterology and ${ }^{2}$ Endoscopy, Fukushima Medical University, \\ School of Medicine, Fukushima 960-1247, Japan
}

Received May 9, 2018; Accepted August 8, 2018

DOI: $10.3892 / \mathrm{mco} .2018 .1696$

\begin{abstract}
Predicting the prognosis of unresectable pancreatic ductal adenocarcinoma (PDAC) is useful in determining the appropriate management strategy. The present study aimed to investigate the association between PDAC prognosis and inflammation-based markers, such as the neutrophil-to-lymphocyte ratio (NLR), platelet-to-lymphocyte ratio, prognostic nutritional index, modified Glasgow prognostic score (mGPS) and controlling nutritional status score. A total of 72 patients with unresectable PDAC who received chemotherapy were included. Inflammation-based markers were measured prior to treatment. The median progression-free survival (PFS) and overall survival (OS) were 117 days (range, 10-781 days) and 244 days (range 43-781 days), respectively. The cut-off value of continuous variables that predicted the median OS (244 days) was calcualted. Univariate analysis of PFS showed that disease stage, first-line chemotherapy regimen, carcinoembryonic antigen (CEA), NLR, platelet-to-lymphocyte ratio (PLR), mGPS and controlling nutritional status (CONUT) scores were associated with PFS. Among them, stage, first-line chemotherapy regimen, CEA, NLR and mGPS were independent
\end{abstract}

Correspondence to: Dr Rei Suzuki, Department of Gastroenterology, Fukushima Medical University, School of Medicine, 1 Hikarigaoka, Fukushima 960-1247, Japan

E-mail: subaru@fmu.ac.jp

Abbreviations: PDAC, pancreatic ductal adenocarcinoma; NLR, neutrophil-to-lymphocyte ratio; PLR, platelet-to-lymphocyte ratio; PNI, prognostic nutritional index; mGPS, modified Glasgow prognostic score; CONUT, controlling nutritional status; PFS, progression-free survival; OS, overall survival; CEA, carcinoembryonic antigen; CA19-9, cancer antigen 19-9; CRP, C-reactive protein

Key words: pancreatic ductal adenocarcinoma, chemotherapy, inflammation-based markers, modified Glasgow prognostic score, predictive factor prognostic factors for PFS in multivariate analysis. Univariate analysis of OS showed that stage, first-line chemotherapy regimen, CA19-9, NLR, PLR, prognostic nutritional index (PNI), mGPS and CONUT score were associated wtih OS. Among them, first-line chemotherapy and mGPS were independent prognostic factors for OS according to multivariate analysis. Univariate and multivariate analyses revealed that a NLR $\geq 4.0$ and mGPS 2 were independent prognostic factors for PFS. For OS, mGPS 2 was an independent prognostic factor. In conclusion, mGPS was the most useful marker in predicting the prognosis of patients with unresectable PDAC who received chemotherapy.

\section{Introduction}

Pancreatic ductal adenocarcinoma (PDAC) is one of the most lethal malignancies worldwide (1). Most PDACs are unresectable at the time of diagnosis and are treated with palliative chemotherapy. Gemcitabine monotherapy was the standard treatment for PDAC until 2011; however, combination chemotherapy regimens such as FOLFIRINOX and gemcitabine plus nab-paclitaxel therapy have become standard treatments in recent years and have improved the prognosis of PDAC patients. However, it is sometimes difficult to determine the most appropriate regimen for each PDAC patient. The prediction of patient prognosis is useful for providing proper treatment.

In advanced cancers, inflammation is associated with tumor proliferative activity. Nutritional state is also important, because patients with advanced cancer tend to become malnourished due to cachexia. Malnutrition leads to poor prognosis and decreased quality of life. Some inflammatory and nutritional scores, such as neutrophil-to-lymphocyte ratio (NLR) (2-4), platelet-to-lymphocyte ratio (PLR) (5), prognostic nutritional index (PNI) (6-8), modified Glasgow prognostic score (mGPS) $(9,10)$, and controlling nutritional status (CONUT) score $(11,12)$, have been reported to be useful in estimating the prognosis of patients with advanced cancers. The definition of each score is shown in Fig. 1. We previously reported that NLR is useful for predicting the outcome of gemcitabine therapy in unresectable PDAC (13). However, 


\begin{tabular}{|c|c|c|c|c|c|}
\hline $\begin{array}{l}\text { NLR = periphe } \\
\text { PLR = periphe }\end{array}$ & absolute neutrophil count d & $d$ by the absc & $\begin{array}{l}\text { mphocyte count } \\
\text { hocyte count }\end{array}$ & & \\
\hline \multicolumn{6}{|c|}{$\mathrm{PNI}=10 \times$ serum albumin concentration $(\mathrm{g} / \mathrm{dl})+0.005 \times$ lymphocytes } \\
\hline \multirow[t]{5}{*}{ mGPS score } & \multicolumn{3}{|l|}{ Biochemical measurements } & \multicolumn{2}{|l|}{ GPS score } \\
\hline & \multicolumn{3}{|c|}{$\mathrm{CRP} \leq 10 \mathrm{mg} / \mathrm{l}$ and albumin $\geq 3.5 \mathrm{~g} / \mathrm{dl}$} & \multicolumn{2}{|l|}{0} \\
\hline & \multicolumn{3}{|c|}{$\mathrm{CRP} \leq 10 \mathrm{mg} / \mathrm{l}$ and albumin $<3.5 \mathrm{~g} / \mathrm{dl}$} & \multicolumn{2}{|l|}{0} \\
\hline & \multicolumn{3}{|c|}{ CRP $>10 \mathrm{mg} / \mathrm{l}$ and albumin $\geq 3.5 \mathrm{~g} / \mathrm{dl}$} & \multicolumn{2}{|l|}{1} \\
\hline & \multicolumn{3}{|c|}{ CRP $>10 \mathrm{mg} / \mathrm{l}$ and albumin $<3.5 \mathrm{~g} / \mathrm{dl}$} & \multicolumn{2}{|l|}{2} \\
\hline \multirow[t]{5}{*}{ CONUT score } & \multicolumn{5}{|l|}{ Parameters } \\
\hline & Albumin (g/dl) & $\geq 3.50[0]$ & $3.00-3.49[2]$ & $2.50-2.99[4]$ & $<2.50[6]$ \\
\hline & Lymphocytes (total/ml ) & $\geq 1600[0]$ & $1200-1599[1]$ & $800-1199$ [2] & $<800[3]$ \\
\hline & Total cholesterol (mg/dl ) & $\geq 180[0]$ & $140-179[1]$ & $100-139[2]$ & $<100[3]$ \\
\hline & Malnutrition status & $\begin{array}{c}\text { Normal } \\
{[0-1 \text { points }]}\end{array}$ & $\begin{array}{c}\text { Mild } \\
\text { [2-4 points] }\end{array}$ & $\begin{array}{l}\text { Moderate } \\
\text { [5-8 points] }\end{array}$ & $\begin{array}{c}\text { Severe } \\
{[>8 \text { points] }}\end{array}$ \\
\hline
\end{tabular}

Figure 1. Definition of inflammatory and nutrition scores.

which marker is the most reliable for predicting the prognosis of PDAC patients treated with chemotherapy, including several combination regimens, remains unknown.

The present study aimed to detect the most useful marker for predicting the prognosis of unresectable PDAC patients treated with chemotherapy.

\section{Materials and methods}

This retrospective observational study reviewed the data of unresectable PDAC patients, including those with locally advanced and metastatic disease, who underwent palliative chemotherapy between September 2006 and August 2016 at Fukushima Medical University Hospital (Fukushima, Japan). All patients were pathologically diagnosed with PDAC, and patients with rare primary pancreatic neoplasms, including acinar cell carcinoma or neuroendocrine carcinoma, were excluded. The chemotherapy regimen (FOLFIRINOX, gemcitabine plus nab-paclitaxel therapy: GnP, gemcitabine, or S-1) was decided by each patient's physician. All clinico-pathological data were measured prior to treatment.

The progression-free survival (PFS) and overall survival (OS) times were calculated from the date of histological diagnosis to the date of disease progression and mortality by any cause, respectively. We applied receiver operating characteristic (ROC) curve analysis to determine ideal cut-off values to predict poor prognosis for the following continuous variables: age, tumor diameter, carcinoembryonic antigen (CEA), cancer antigen 19-9 (CA19-9), NLR, PLR, PNI and CONUT score. The association of each clinico-pathological parameter (age, gender, tumor diameter, disease stage (UICC7th), first-line chemotherapy, NLR, PLR, PNI, mGPS and CONUT score) with PFS and OS time was investigated. Survival analysis was performed using the Kaplan-Meier method with log-rank tests in univariate analysis. Multivariate Cox regression analysis was performed to determine the effect of clinico-pathological variables on survival time. The data were analyzed using EZR (Saitama Medical Center, Jichi Medical University, Saitama, Japan), a graphical user interface for R (The R Foundation for Statistical Computing, version 3.2.1) that was a modified version of the $\mathrm{R}$ commander (version 2.1-7). Differences with a $P$-value $<0.05$ were considered statistically significant (14). This study was approved by the Institutional Review Board of Fukushima Medical University.

\section{Results}

We included 72 PDAC patients who met the inclusion criteria described above in the analysis. The characteristics and laboratory data of the 72 patients are shown in Table I. The median PFS and OS was 117 days (range, 10-781 days) and 244 days (range, 43-781 days), respectively.

We calculated the cut-off value of continuous variables that predicted the median OS (244 days) using ROC analysis. The cut-off value of laboratory data and inflammatory markers are shown in Table II.

Univariate analysis of PFS showed that disease stage (locally advanced vs. metastatic, $\mathrm{P}<0.001)$, first-line chemotherapy regimen (combination therapy vs. monotherapy, $\mathrm{P}<0.001$ ), CEA ( $<5.0$ vs. $\geq 5.0 \mathrm{ng} / \mathrm{ml}, \mathrm{P}=0.043), \operatorname{NLR}(<4.0$ vs. $\geq 4.0$, $\mathrm{P}<0.001)$, PLR (182.0< vs. $\geq 182.0, \mathrm{P}=0.044)$, mGPS (0, 1 vs. 2 , $\mathrm{P}<0.001)$ and CONUT score $(3<\mathrm{vs} . \geq 3, \mathrm{P}=0.034)$ were related to PFS. Among them, stage $(\mathrm{P}=0.036)$, first-line chemotherapy regimen $(\mathrm{P}<0.001)$, CEA $(\mathrm{P}=0.024)$, NLR $(\mathrm{P}<0.001)$ and mGPS $(\mathrm{P}=0.005)$ were independent prognostic factors for PFS in multivariate analysis (Table III). 
Table I. Patient characteristics.

\begin{tabular}{|c|c|}
\hline Category & Value \\
\hline Age, years ${ }^{a}$ & $63(42-85)$ \\
\hline \multicolumn{2}{|l|}{$\operatorname{Sex}^{\mathrm{b}}$} \\
\hline Male & $40(55.6 \%)$ \\
\hline Female & $32(44.4 \%)$ \\
\hline Tumor diameter, $\mathrm{mm}^{\mathrm{a}}$ & $30(7-106)$ \\
\hline \multicolumn{2}{|l|}{ Stage $^{b}$} \\
\hline Locally advanced & $20(27.8 \%)$ \\
\hline Metastatic & $52(72.2 \%)$ \\
\hline \multicolumn{2}{|l|}{ First-line regimen $^{\mathrm{b}}$} \\
\hline GEM + nab-PTX & $16(22.2 \%)$ \\
\hline FOLFIRINOX & $10(13.9 \%)$ \\
\hline GEM & $44(61.1 \%)$ \\
\hline S-1 & $2(2.8 \%)$ \\
\hline $\mathrm{CEA}(\mathrm{ng} / \mathrm{ml})^{\mathrm{a}}$ & $4.2(1.2-1738.0)$ \\
\hline CA19-9 (U/ml $)^{\mathrm{a}}$ & $1326.1(0.3-603000.2)$ \\
\hline $\mathrm{NLR}^{\mathrm{a}}$ & $3.2(1.2-11.6)$ \\
\hline $\mathrm{PLR}^{\mathrm{a}}$ & $175.0(63.9-547.1)$ \\
\hline $\mathrm{PNI}^{\mathrm{a}}$ & $45.5(32.4-60.7)$ \\
\hline \multicolumn{2}{|l|}{$\mathrm{mGPS}^{\mathrm{b}}$} \\
\hline 0 & $52(72.2 \%)$ \\
\hline 1 & $11(15.3 \%)$ \\
\hline 2 & $9(12.5 \%)$ \\
\hline \multicolumn{2}{|l|}{ CONUT score ${ }^{\mathrm{b}}$} \\
\hline $0-1$ & $18(27.3 \%)$ \\
\hline $2-4$ & $39(59.1 \%)$ \\
\hline $5-8$ & $8(12.1 \%)$ \\
\hline $8<$ & $1(1.5 \%)$ \\
\hline
\end{tabular}

aData presented as the mean (range); ${ }^{b}$ Data presented as the number of patients (\%). GEM, gemcitabine; nab-PTX, nab-paclitaxel; CEA, carcinoembryonic antigen; CA19-9, cancer antigen 19-9; NLR, neutrophil-to-lymphocyte ratio; PLR, platelet-to-lymphocyte ratio; PNI, prognostic nutritional index; mGPS, modified Glasgow prognostic score; CONUT, controlling nutritional status.

Univariate analysis of OS showed that the stage $(\mathrm{P}<0.001)$, first-line chemotherapy regimen $(\mathrm{P}<0.001), \mathrm{CA19-9}(\mathrm{P}=0.040)$, NLR $(\mathrm{P}<0.001)$, PLR ( $\mathrm{P}=0.004)$, PNI (<45.2 vs. $\geq 45.2$, $\mathrm{P}=0.013), \mathrm{mGPS}(\mathrm{P}<0.001)$ and CONUT score $(\mathrm{P}=0.016)$ were related to OS. Among them, first-line chemotherapy $(\mathrm{P}=0.007)$ and mGPS $(\mathrm{P}<0.001)$ were independent prognostic factors for OS according to multivariate analysis (Table IV).

The Kaplan-Meier analysis of mGPS revealed that the survival time of patients with mGPS 2 was significantly poorer than that of patients with mGPS 0 and 1 (Figs. 2 and 3).

\section{Discussion}

We aimed to clarify the best inflammation-based marker to predict the prognosis of unresectable PDAC patients treated with chemotherapy. The results showed that mGPS was the most reliable inflammation-related marker to predict PFS and
Table II. Diagnostic yield of each marker at the optimal cut-off values.

\begin{tabular}{lcccc}
\hline Variable & $\begin{array}{c}\text { Cut-off } \\
\text { value }\end{array}$ & Sensitivity & Specificity & Accuracy \\
\hline Age, years & $<64$ & 52.8 & 63.9 & 55.0 \\
Size, $\mathrm{mm}$ & $<28$ & 41.7 & 66.7 & 53.2 \\
CEA, ng/ml & $<5.0$ & 63.9 & 44.4 & 49.5 \\
CA19-9, U/ml & $<1772$ & 61.1 & 50.0 & 52.0 \\
NLR & $<4.0$ & 75.0 & 55.6 & 63.3 \\
PLR & $<182.0$ & 63.9 & 58.3 & 60.1 \\
PNI & $\geq 45.2$ & 63.9 & 55.6 & 54.2 \\
CONUT score & $<3$ & 70.6 & 43.8 & 61.7 \\
\hline
\end{tabular}

CEA, carcinoembryonic antigen; CA19-9, cancer antigen 19-9; NLR, neutrophil-to-lymphocyte ratio; PLR, platelet-to-lymphocyte ratio; PNI, prognostic nutritional index; mGPS, modified Glasgow prognostic score; CONUT, controlling nutritional status.

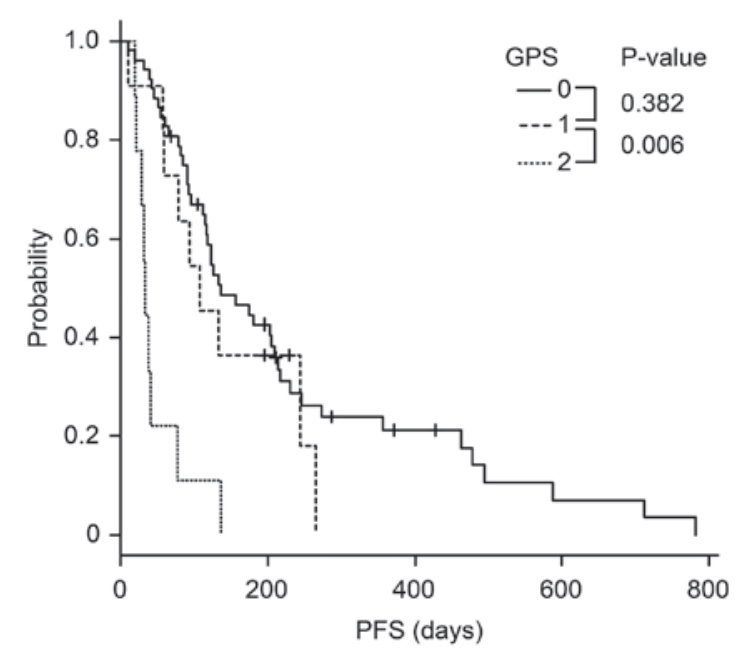

Figure 2. Kaplan-Meier analysis of progression-free survival according to mGPS. The PFS time of patients with mGPS 2 was significantly poorer than that of patients with mGPS 0 and 1 .

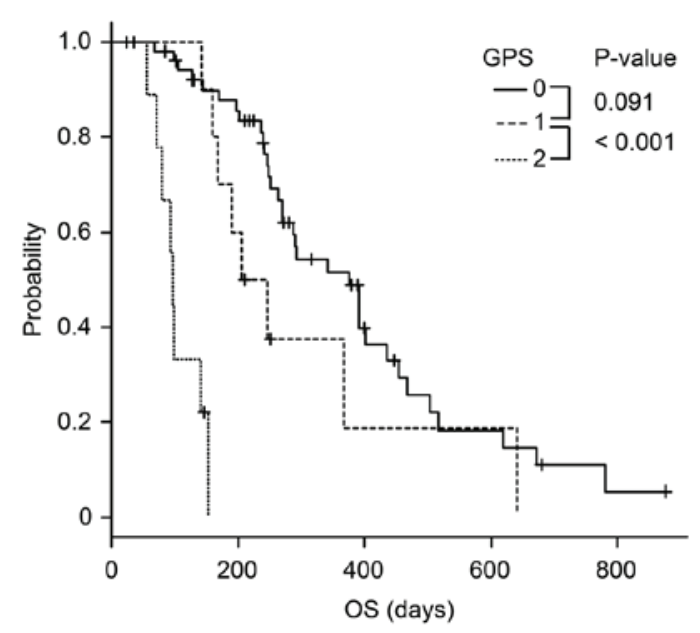

Figure 3. Kaplan-Meier analysis of overall survival according to mGPS. The OS of patients with mGPS 2 was significantly poorer than that of patients with mGPS 0 and 1. 
Table III. Univariate and multivariate analyses to detect independent prognostic factors for PFS.

\begin{tabular}{|c|c|c|c|c|c|}
\hline \multirow[b]{2}{*}{ Variable } & \multirow[b]{2}{*}{ No. } & \multicolumn{2}{|c|}{ Univariate } & \multicolumn{2}{|c|}{ Multivariate } \\
\hline & & Median survival, days & P-value & $\operatorname{HR}(95 \% \mathrm{CI})$ & P-value \\
\hline Age, years & & & 0.302 & & \\
\hline$<64$ & 32 & 136 & & & \\
\hline$\geq 64$ & 40 & 108 & & & \\
\hline Sex & & & 0.625 & & \\
\hline Male & 40 & 123 & & & \\
\hline Female & 32 & 118 & & & \\
\hline Diameter, mm & & & 0.465 & & \\
\hline$<28$ & 27 & 134 & & & \\
\hline$\geq 28$ & 45 & 117 & & & \\
\hline Stage & & & $<0.001$ & $2.36(1.06-5.06)$ & 0.036 \\
\hline Locally advanced & 20 & 244 & & & \\
\hline Metastatic & 52 & 94 & & & \\
\hline First-line regimen & & & $<0.001$ & $0.25(0.12-0.53)$ & $<0.001$ \\
\hline Combination therapy & 24 & 244 & & & \\
\hline Monotherapy & 48 & 92 & & & \\
\hline CEA, ng/ml & & & 0.043 & $2.24(1.11-4.53)$ & 0.024 \\
\hline$<5.0$ & 43 & 136 & & & \\
\hline$\geq 5.0$ & 29 & 115 & & & \\
\hline CA19-9, U/ml & & & 0.106 & $0.89(0.42-1.88)$ & 0.765 \\
\hline$<1,772$ & 40 & 147 & & & \\
\hline$\geq 1,772$ & 32 & 108 & & & \\
\hline NLR & & & $<0.001$ & $6.93(2.77-17.35)$ & $<0.001$ \\
\hline$<4.0$ & 43 & 203 & & & \\
\hline$\geq 4.0$ & 29 & 77 & & & \\
\hline PLR & & & 0.044 & $0.47(0.22-1.00)$ & 0.050 \\
\hline$<182.0$ & 38 & 156 & & & \\
\hline$\geq 182.0$ & 34 & 92 & & & \\
\hline PNI & & & 0.113 & $1.09(0.55-2.18)$ & 0.807 \\
\hline$<45.2$ & 39 & 112 & & & \\
\hline$\geq 45.2$ & 33 & 126 & & & \\
\hline mGPS & & & $<0.001$ & $5.00(1.64-15.23)$ & 0.005 \\
\hline 0,1 & 63 & 134 & & & \\
\hline 2 & 9 & 33 & & & \\
\hline CONUT score & & & 0.034 & $0.87(0.41-1.84)$ & 0.722 \\
\hline$<3$ & 42 & 156 & & & \\
\hline$\geq 3$ & 24 & 89 & & & \\
\hline
\end{tabular}

PFS, progression-free survival; HR, hazard ratio; CEA, carcinoembryonic antigen; CA19-9, cancer antigen 19-9; NLR, neutrophil-to-lymphocyte ratio; PLR, platelet-to-lymphocyte ratio; PNI, prognostic nutritional index; mGPS, modified Glasgow prognostic score; CONUT, controlling nutritional status.

OS. To the best of our knowledge, this study is the first to clarify the most useful inflammatory marker to predict prognosis in cases of unresectable PDAC treated with palliative chemotherapy.

Several studies have assessed the association between inflammation-based markers and the prognosis of resectable and unresectable PDAC patients. In patients with resectable PDAC, preoperative NLR (15), PLR (16,17), GPS (18) and PNI 45 (7) were reported to be factors in early recurrence and poor prognosis after surgical resection. In unresectable PDAC, previous reports showed that patients with a high NLR had a shorter OS time $(13,19)$. In terms of GPS, a retrospective study 
Table IV. Univariate and multivariate analyses to detect independent prognostic factors for OS.

\begin{tabular}{|c|c|c|c|c|c|}
\hline \multirow[b]{2}{*}{ Variable } & \multirow[b]{2}{*}{ No. } & \multicolumn{2}{|c|}{ Univariate } & \multicolumn{2}{|c|}{ Multivariate } \\
\hline & & Median survival, days & P-value & $\operatorname{HR}(95 \% \mathrm{CI})$ & P-value \\
\hline Age, years & & & 0.073 & & \\
\hline$<64$ & 32 & 292 & & & \\
\hline$\geq 64$ & 40 & 271 & & & \\
\hline $\operatorname{Sex}$ & & & 0.801 & & \\
\hline Male & 40 & 270 & & & \\
\hline Female & 32 & 292 & & & \\
\hline Diameter, mm & & & 0.399 & & \\
\hline$<28$ & 27 & 391 & & & \\
\hline$\geq 28$ & 45 & 251 & & & \\
\hline Stage & & & $<0.001$ & $2.31(0.91-5.90)$ & 0.078 \\
\hline Locally advanced & 20 & 641 & & & \\
\hline Metastatic & 52 & 249 & & & \\
\hline First-line regimen & & & $<0.001$ & $0.25(0.09-0.69)$ & 0.007 \\
\hline Combination therapy & 24 & 641 & & & \\
\hline Monotherapy & 48 & 241 & & & \\
\hline $\mathrm{CEA}, \mathrm{ng} / \mathrm{ml}$ & & & 0.268 & $1.19(0.52-2.73)$ & 0.680 \\
\hline$<5.0$ & 43 & 292 & & & \\
\hline$\geq 5.0$ & 29 & 251 & & & \\
\hline CA19-9, U/ml & & & 0.040 & $1.67(0.68-4.11)$ & 0.263 \\
\hline$<1,772$ & 40 & 376 & & & \\
\hline$\geq 1,772$ & 32 & 247 & & & \\
\hline NLR & & & $<0.001$ & $2.18(0.88-5.38)$ & 0.092 \\
\hline$<4.0$ & 43 & 392 & & & \\
\hline$\geq 4.0$ & 29 & 206 & & & \\
\hline PLR & & & 0.004 & $0.99(0.47-2.10)$ & 0.984 \\
\hline$<182.0$ & 38 & 391 & & & \\
\hline$\geq 182.0$ & 34 & 236 & & & \\
\hline PNI & & & 0.013 & $0.52(0.23-1.19)$ & 0.123 \\
\hline$<45.2$ & 39 & 249 & & & \\
\hline$\geq 45.2$ & 33 & 376 & & & \\
\hline mGPS & & & $<0.001$ & $26.16(5.22-131.10)$ & $<0.001$ \\
\hline 0,1 & 63 & 342 & & & \\
\hline 2 & 9 & 97 & & & \\
\hline CONUT score & & & 0.016 & $0.69(0.29-1.67)$ & 0.413 \\
\hline$<3$ & 42 & 368 & & & \\
\hline$\geq 3$ & 24 & 249 & & & \\
\hline
\end{tabular}

OS, overall survival; HR, hazard ratio; CEA, carcinoembryonic antigen; CA19-9, cancer antigen 19-9; NLR, neutrophil-to-lymphocyte ratio; PLR, platelet-to-lymphocyte ratio; PNI, prognostic nutritional index; mGPS, modified Glasgow prognostic score; CONUT, controlling nutritional status.

intended for PDAC patients treated with gemcitabine showed that GPS could predict poor prognosis (20). PNI has been shown to be related to prognosis and a systemic inflammatory response (i.e., NLR, PLR and serum TNF- $\alpha$ level) in PDAC (8).

There are many reports concerning the relation between each inflammation-based marker and the prognosis of
PDAC, but there are few reports comparing the utility of several inflammation-based markers. Yamada et al (21) conducted a retrospective study to clarify which score could best reflect survival in resected pancreatic cancer patients and found that GPS was superior to the other markers. Additionally, a systematic review summarized past reports 
about PDAC and inflammatory markers and concluded that GPS and NLR were useful in predicting prognosis (22); however, unlike our study, this study included patients with various stages and treatments (e.g., surgical resection and chemotherapy).

mGPS, which most accurately reflected the prognosis of unresectable PDAC patients in our study, is based on C-reactive protein (CRP) and serum albumin levels. CRP elevation indicates systemic inflammation. Because the cytokines produced by tumors increase the inflammatory response, CRP is considered to reflect growth activity in tumors. Furthermore, the serum albumin level indicates patient nourishment state and is related to performance status. Unresectable PDAC patients often fall into an undernourished state because of a decrease in pancreatic exocrine function and cancer cachexia. This undernourished state worsens the prognosis by reducing the patient's quality of life and making the continuation of chemotherapy more difficult. In the original GPS, hypoalbuminemia (Alb $<3.5 \mathrm{~g} / \mathrm{dl}$ ) or elevated C-reactive protein (CRP $>10 \mathrm{mg} / \mathrm{l}$ ) is defined as a score of 1 (23). The modified GPS, which defined hypoalbuminemia without elevated CRP as a score of 0 , was proposed because the GPS score of 1 was most commonly due to elevated CRP (24). In this study, mGPS 2 patients had a significantly poorer prognosis than those with scores of 0 or 1 because of the high-grade malignancy and cachexic state of the tumors. Patients with a score of 1 tended to have a poorer prognosis than patients with a score of 0 , but the difference was not significant. This result indicates that nourishment state plays a very important role in determining the prognosis of PDAC. Naturally, serum CRP levels can increase as a result of an infection rather than tumor activity. Therefore, we used blood data collected in the afebrile state just before the initiation of chemotherapy, but some cases might be complicated by occult cholangitis.

A limitation of this study was that it was a single-center study with a limited number of patients. The results should be validated in a large population across multiple clinical sites. In addition, the chemotherapy regimen was determined at the physician's discretion, which may have resulted in bias. Studies focused only on patients treated with the single regimen or on those treated with multidrug therapy are expected.

In conclusion, mGPS appears to be an independent prognostic factor for predicting the PFS and OS of unresectable PDAC patients. Predicting prognosis before the start of therapy may be helpful in choosing the optimal treatment.

\section{Acknowledgements}

We thank all of the staff members of the Department of Gastroenterology of Fukushima Medical University, the Department of Endoscopy of Fukushima Medical University Hospital, and the gastroenterology ward of Fukushima Medical University Hospital. We also thank American Journal Experts for their English editing services.

\section{Funding}

Supported by Department of Gastroenterology, Fukushima Medical University, School of Medicine.

\section{Availability of data and materials}

The datasets obtained and/or analyzed during the current study are available from the corresponding author on reasonable request.

\section{Authors' contributions}

HA and RS designed and performed the research; HA, RS and $\mathrm{HO}$ analyzed the data; HA, RS and HO wrote the manuscript; TH, TT, MS, NK, HT, KW, JN, HK, MT, YS and HI provided clinical advice; $\mathrm{TH}$ and $\mathrm{HO}$ supervised the report.

\section{Ethics approval and consent to participate}

This study was reviewed and approved by the Ethics Committee of the Fukushima Medical University Hospital. Patients were not required to provide informed consent for this study because the analysis used anonymous clinical data that were obtained after each patient agreed to treatment by written consent.

\section{Patient consent for publication}

Patients were not required to provide informed consent for this study because the analysis used anonymous clinical data that were obtained after each patient agreed to treatment by written consent. For full disclosure, the details of the study are published on the home page of Fukushima Medical University.

\section{Competing interests}

The authors declare that they have no competing interests.

\section{References}

1. Siegel RL, Miller KD and Jemal A: Cancer statistics, 2016. CA Cancer J Clin 66: 7-30, 2016.

2. Zahorec R: Ratio of neutrophil to lymphocyte counts-rapid and simple parameter of systemic inflammation and stress in critically ill. Bratisl Lek Listy 102: 5-14, 2001 (In English, Slovak).

3. Walsh SR, Cook EJ, Goulder F, Justin TA and Keeling NJ: Neutrophil-lymphocyte ratio as a prognostic factor in colorectal cancer. J Surg Oncol 91: 181-184, 2005.

4. An X, Ding PR, Li YH, Wang FH, Shi YX, Wang ZQ, He YJ, $\mathrm{Xu} \mathrm{RH}$ and Jiang WQ: Elevated neutrophil to lymphocyte ratio predicts survival in advanced pancreatic cancer. Biomarkers 15: 516-522, 2010.

5. Aliustaoglu M, Bilici A, Seker M, Dane F, Gocun M, Konya V, Ustaalioglu BB and Gumus M: The association of pre-treatment peripheral blood markers with survival in patients with pancreatic cancer. Hepatogastroenterology 57: 640-645, 2010

6. Lowe EF, Stein M, Woolley T, Waycaster M, Scroggins B, Acuff R, Smith JT and Lefemine AA: Prognostic nutritional index: Its usefulness as a predictor of clinical course. J Am Coll Nutr 2: 231-240, 1983

7. Kanda M, Fujii T, Kodera Y, Nagai S, Takeda S and Nakao A: Nutritional predictors of postoperative outcome in pancreatic cancer. Br J Surg 98: 268-274, 2011.

8. Geng Y, Qi Q, Sun M, Chen H, Wang P and Chen Z: Prognostic nutritional index predicts survival and correlates with systemic inflammatory response in advanced pancreatic cancer. Eur J Surg Oncol 41: 1508-1514, 2015.

9. Glen P, Jamieson NB, McMillan DC, Carter R, Imrie CW and McKay CJ: Evaluation of an inflammation-based prognostic score in patients with inoperable pancreatic cancer. Pancreatology 6: 450-453, 2006 
10. Imaoka H, Mizuno N, Hara K, Hijioka S, Tajika M, Tanaka T, Ishihara M, Yogi T, Tsutsumi H, Fujiyoshi T, et al: Evaluation of modified glasgow prognostic score for pancreatic cancer: A retrospective cohort study. Pancreas 45: 211-217, 2016.

11. Ignacio de Ulíbarri J, González-Madroño A, de Villar NG, González P, González B, Mancha A, RodríguezF and Fernández G: CONUT: A tool for controlling nutritional status. First validation in a hospital population. Nutr Hosp 20: 38-45, 2005.

12. Iseki Y, Shibutani M, Maeda K, Nagahara H, Ohtani H, Sugano K, Ikeya T, Muguruma K, Tanaka H, Toyokawa T, et al: Impact of the preoperative controlling nutritional status (CONUT) score on the survival after curative surgery for colorectal cancer. PLoS One 10: e0132488, 2015.

13. Suzuki R, Takagi T, Hikichi T, Konno N, Sugimoto M, Watanabe KO, Nakamura J, Waragai Y, Kikuchi H, Takasumi M, et al: Derived neutrophil/lymphocyte ratio predicts gemcitabine therapy outcome in unresectable pancreatic cancer. Oncol Lett 11: 3441-3445, 2016.

14. Kanda Y: Investigation of the freely available easy-to-use software 'EZR' for medical statistics. Bone Marrow Transplant 48 : 452-458, 2013

15. Garcea G, Ladwa N, Neal CP, Metcalfe MS, Dennison AR and Berry DP: Preoperative neutrophil-to-lymphocyte ratio (NLR) is associated with reduced disease-free survival following curative resection of pancreatic adenocarcinoma. World J Surg 35 868-872, 2011.

16. Song W, Tian C, Wang K, Zhang RJ and Zou SB: Preoperative platelet lymphocyte ratio as independent predictors of prognosis in pancreatic cancer: A systematic review and meta-analysis. PLoS One 12: e0178762, 2017.

17. Shirai Y, Shiba H, Sakamoto T, Horiuchi T, Haruki K, Fujiwara Y, Futagawa Y, Ohashi T and Yanaga K: Preoperative platelet to lymphocyte ratio predicts outcome of patients with pancreatic ductal adenocarcinoma after pancreatic resection. Surgery 158 : $360-365,2015$
18. Morinaga S, Murakawa M, Katayama Y, Yamaoku K, Aoyama T, Kanazawa A, Higuchi A, Shiozawa M, Kobayashi S, Ueno M and Morimoto M: Glasgow prognostic score predicts clinical outcomes in patients with pancreatic cancer undergoing adjuvant gemcitabine monotherapy after curative surgery. Anticancer Res 35: 4865-4870, 2015

19. Xue P, Kanai M, Mori Y, Nishimura T, Uza N, Kodama Y, Kawaguchi Y, Takaori K, Matsumoto S, Uemoto S and Chiba T: Neutrophil-to-lymphocyte ratio for predicting palliative chemotherapy outcomes in advanced pancreatic cancer patients. Cancer Med 3: 406-415, 2014

20. Shimoda M, Katoh M, Kita J, Sawada T and Kubota K: The glasgow prognostic score is a good predictor of treatment outcome in patients with unresectable pancreatic cancer. Chemotherapy 56: 501-506, 2010.

21. Yamada S, Fujii T, Yabusaki N, Murotani K, Iwata N, Kanda M, Tanaka C, Nakayama G, Sugimoto H, Koike M, et al: Clinical implication of inflammation-based prognostic score in pancreatic cancer: Glasgow prognostic score is the most reliable parameter. Medicine (Baltimore) 95: e3582, 2016.

22. Ahmad J, Grimes N, Farid S and Morris-Stiff G: Inflammatory response related scoring systems in assessing the prognosis of patients with pancreatic ductal adenocarcinoma: A systematic review. Hepatobiliary Pancreat Dis Int 13: 474-481, 2014.

23. Forrest LM, McMillan DC, McArdle CS, Angerson WJ, Dagg K and Scott HR: A prospective longitudinal study of performance status, an inflammation-based score (GPS) and survival in patients with inoperable non-small-cell lung cancer. Br J Cancer 92: 1834-1836, 2005.

24. McMillan DC, Crozier JE, Canna K, Angerson WJ and McArdle CS: Evaluation of an inflammation-based prognostic score (GPS) in patients undergoing resection for colon and rectal cancer. Int J Colorectal Dis 22: 881-886, 2007. 